\title{
The Use of Causal Language and Filled Pauses in Children with and without Autism
}

\author{
Anna Eva Hallin, ${ }^{1}$ Gabrielle D. Garcia, ${ }^{2,3}$ and Christina Reuterskiöld ${ }^{1}$ \\ ${ }^{1}$ Department of Communicative Sciences and Disorders, New York University, 665 Broadway, 9th Floor, \\ New York, NY 10012, USA \\ ${ }^{2}$ Department of Communication Sciences and Disorders, Northwestern University, 70 Arts Circle Drive, \\ Evanston, IL 60208, USA \\ ${ }^{3}$ Department of Psychology, New York University Abu Dhabi, P.O. Box 129188, Saadiyat Island, Abu Dhabi, UAE
}

Correspondence should be addressed to Anna Eva Hallin; ae.hallin@nyu.edu

Received 27 April 2016; Accepted 24 August 2016

Academic Editor: Anna L. Theakston

Copyright (C) 2016 Anna Eva Hallin et al. This is an open access article distributed under the Creative Commons Attribution License, which permits unrestricted use, distribution, and reproduction in any medium, provided the original work is properly cited.

\begin{abstract}
This study investigated the relationship between pragmatic ability and two aspects of structural language in conversational language samples from 24 school-age children with and without high-functioning autism (HFA): causal statements and speech disruptions. In contrast to a majority of previous studies, grammatical complexity and mean length of utterance were factored into the analyses, since these are potential confounding variables. The results showed that children with HFA used fewer spontaneous causal statements and fewer filled pauses in conversation compared to children with typical development (TD). There was also a significant and positive relationship between filled pauses and pragmatic ability after controlling for structural language ability. The results may help us understand the conversational patterns of children with HFA better.
\end{abstract}

\section{Introduction}

Language sample analysis (LSA) provides the opportunity to study a child's language skills in a naturalistic context (e.g., conversation or narratives) and many researchers have advocated for LSA as a substitute or complement to standardized testing of children with communication difficulties [13]. Standard LSA measures include mean length of utterance (MLU) and syntactic complexity and grammatical errors, and these measures have been shown to capture differences between children and adolescents with specific language impairment (SLI) and children with typical development (TD) [4-6]. While children with SLI, who primarily show difficulties with aspects of language form, might find some types of discourse (e.g., narratives or expository tasks) challenging due to the increased demands on their structural language skills (e.g., $[4,7]$ ), children with difficulties mainly in the social domain might be more challenged by pragmatic demands such as turn-taking management, which may not be captured by standard LSA measures (cf. $[8,9]$ ).

In the current study, conversational language samples from school-age children with high-functioning autism (HFA) and their TD peers are analyzed. Children with HFA score within normal range on standardized measures of structural language and nonverbal intelligence but show communication difficulties originating from pragmatic difficulties. This group comprises a small part of all individuals with autism $(<5 \%)$ [10], but the relationship between pragmatic difficulties and characteristics of language production can be examined in analyzing language samples from these children. Conversation has been shown to be especially challenging for children and adolescents with HFA due to the pragmatic demands of this context [11-13] and is therefore chosen as a sampling context in the current study. Two LSA measures are in focus: causal statements (clauses beginning with because or so, hypothesized to be related to the ability to gauge listener needs and provide explanations and details) 
and filled pauses (hypothesized to be related to turn-taking skills). Pragmatic skills are measured with the standardized parent questionnaire, the Children's Communication Checklist-2 (CCC-2) [14]. The items in the CCC-2 target a broad range of pragmatic behaviors in everyday settings and have been found to capture pragmatic impairment in children with HFA better than formal standardized tests $[15,16]$. By using a clinically available tool for LSA (SALT [17]) and choosing measures that are easy to code and quantify, the results will have practical and clinical applications in working with children with pragmatic difficulties and understanding their conversational patterns.

As mentioned previously, children with HFA are not expected to show differences in many of the standard LSA measures that have been found to differentiate children with SLI from TD peers. Several studies have confirmed this: if individuals on the autism spectrum are matched with TD peers on structural language skills, there are no significant differences between groups in, for example, overall grammatical complexity $[18,19]$, tense use [20], or length of narratives [21-23]. This notion was further highlighted by Norbury and Bishop [22], who found that linguistic competence was the strongest influencing factor on narrative performance in children with HFA, SLI, and pragmatic language impairment, not diagnostic status or pragmatic competence. It is therefore essential to take linguistic skills into account when investigating language sample features that might be related to pragmatic ability. Previous studies that have related LSA measures and measures of pragmatic ability, theory of mind, or severity of autism symptomology have generally failed to control for or report structural language measures, which limits the conclusions that can be drawn $[18,21,24]$.

The first aim of the present study is to investigate the relationship between pragmatic ability and causal statements in conversation, while controlling for grammatical complexity. Causal statements are a measure of interest since limited use can be interpreted from a pragmatic perspective, in that the underlying reason could be a lack of sensitivity to listener interests and needs. For example, when a child says "I like ice cream, because I can choose different flavors," the statement spontaneously provides information about the child's own beliefs that would otherwise remain inaccessible to the listener. The child also follows the Western-European social communicative convention of adding an explanation after a mental state has been provided [25, p. 697]. Importantly, however, the use of causal statements can also be related to structural language development. Causal statements are often complex sentences, and causal complex sentences with the connectives "because" or "so" are developmentally late emerging forms (see, e.g., [26]).

Causal language has mainly been explored in pictureelicited narratives from children and adults with HFA and not in conversation. A conversation places a set of different pragmatic demands on the participant in terms of both speaking and listening, such as turn-taking and topic management [11-13]. Existing work has also typically neglected examining the syntactic complexity of the speech samples. The results are inconclusive: some have found that children with HFA use less causal language than children with TD [18, 21, 23, 27, 28], and some have not found any group differences [22, 29-32]. One reason for this could be varying methods and definitions. In some studies, causal statements have been divided into those that explain mental states, actions, or behaviors, including both concrete and inferred causal statements [18, 21, 22, 27]. Other studies include passive constructions and causal verb phrases as well ("made...happen") [29] or an even broader definition of causal language involving narrative cohesion [23]. For the purpose of this study, any use of the connectives "because" or "so" is coded as causal language (similar to Tager-Flusberg and Sullivan [31] and Suh et al. [32]). The reason for this is simplicity: a plain computerized search of a transcript can yield all instances of causal language, which increases reliability. In addition, the simplicity of the measure makes it more clinically useful, if group differences are found.

The second aim of the present study is to investigate speech disruptions in conversation and their relationship to pragmatic ability, while explicitly reporting and factoring in utterance length in the analyses. Speech disruptions, or mazes, include revisions or self-repairs, self-repetitions, and filled pauses (e.g., uh, um, and filler words such as like or well). Speech disruptions are most often studied from a language processing perspective (e.g., $[33,34])$, and the number of speech disruptions typically increases when grammatical complexity and utterance length increase (e.g., $[5,7,35])$. In addition, it has been argued that some speech disruptions could serve a pragmatic function. For example, filled pauses may act as turn-taking devices signaling that the speaker is not finished with his or her turn and/or as verbal markers signaling that a production problem is about to happen (e.g., [36-39]).

Studies analyzing conversational samples from children with TD and HFA have found that children with HFA exhibit fewer filled pauses compared to their peers, but these studies have failed to report MLU or grammatical complexity, which means that the results could be a consequence of less complex language rather than pragmatic difficulties [40-42]. Lake et al. [38] found that adults with HFA produced significantly fewer self-corrections and filled pauses than a TD group in conversation but more repetitions and long $(>2 \mathrm{~s})$ silent pauses. The authors noted that the groups also differed significantly in MLU (TD > HFA) but pointed out that the distribution of the different speech disruptions differed between groups, even when MLU values overlapped. Suh et al. [32] matched adolescents with HFA and TD (1016 years old) on MLU in a narrative task and found that adolescents with HFA had significantly more repetitions and self-corrections than the comparison group. The difference in fillers, where HFA participants had fewer fillers than children with TD, failed to reach significance $(p=.07)$, however, possibly due to lack of power. The narrative context, which provides less need to hold your turn with a filled pause compared to a conversational context, might also have contributed to the nonsignificance. These two latter studies provide some substance to the claim that individuals with HFA show fewer fillers due to pragmatic difficulties rather than shorter utterances, but the results are still inconclusive. 
The direct relationship between pragmatic ability and speech disruptions has only been explored in one previous study [24], where the authors found that the use of the filled pause $u m$ in a brief picture description task was correlated with Social Communication Questionnaire scores in individuals with ASD (8-21 years old). In this study, higher autism symptomology was associated with fewer ums. A similar relationship was not found for $u h$, and no significant relationships were found in their TD group. There was a potential confounding factor in utterance length, however, in that the authors did not control for MLU. In addition the significant relationship seemed to be driven by only six of the participants with ASD, which makes the evidence weaker. The direct relationship between different types of speech disruptions and pragmatic ability, while also controlling for MLU and/or structural language ability, has not been investigated previously to the authors' knowledge.

In summary, previous studies are inconclusive, and it remains uncertain whether children with HFA show less use of causal language and filled pauses due to their pragmatic difficulties or due to overall less complex language in their language samples. Therefore, the current study will analyze conversational language samples and relate the severity of pragmatic difficulties to causal language and speech disruptions use, while taking language complexity into account. This study is guided by the following questions and corresponding hypotheses:

(1) Do two groups of children that vary in their pragmatic skills but are matched on structural language skills (children with HFA and children with TD) differ in terms of

(a) their spontaneous use of causal language in conversation?

(b) the proportion of filled pauses in conversation?

(2) While taking structural language ability into account, is pragmatic ability (measured by the CCC-2) associated with

(a) the proportion of causal statements in conversation?

(b) the proportion of filled pauses and repetition/revisions in conversation?

Children with HFA are predicted to show a significantly smaller proportion of spontaneous causal language use and fewer filled pauses in conversation compared to their TD peers based on the hypothesized pragmatic functions of causal statements and filled pauses (satisfying listener needs of details and explanations and holding the floor between speaker turns, resp.). The groups are not predicted to differ in their grammatical complexity or in their proportion of revisions and repetitions. In addition, pragmatic ability is predicted to be positively and significantly correlated with independent causal statements and filled pauses but not correlated with repetitions and revisions.

\section{Method}

Informed oral consent to participate in the study was obtained from all participating children, and written consent was obtained from the caregivers according to a protocol approved by the New York University Committee on Activities Involving Human Subjects.

2.1. Participants. Seven children with HFA (2 girls, 5 boys) and 17 children with TD (12 girls, 5 boys) were recruited from the same public schools in the New York area, both from regular classrooms and inclusive classrooms for children with high-functioning autism (the Nest Program). The Nest Program follows the normal academic curriculum in integrated classrooms with fewer students and more adult support. The students are given additional instructional and behavioral support, as well as regular speech-language therapy.

All children were in the second or third grade (ages $7: 1$ to $9: 2$ ). Children with TD had no history or signs of developmental or academic delays according to parents and teachers. Children with HFA had all received a diagnosis of an autism spectrum disorder (as determined by the ADOS [43]). Two of the children with HFA also had a diagnosis of ADHD, which is common in children with HFA [44]. English was the primary language for all children according to the parent questionnaire or as confirmed by the classroom teacher. All children passed pure tone audiometric screening at $30 \mathrm{~dB}$ bilaterally at $1000-4000 \mathrm{~Hz}$.

\subsection{Standardized Measures}

2.2.1. Verbal and Nonverbal Tests. All children were given the Test of Nonverbal Intelligence (TONI, 3rd Edition) [45] to ensure that their nonverbal ability was within the normal range (i.e., a standard score of 85 or above). They were also given an age-appropriate Test of Language Development (TOLD) version (i.e., for children 7:0-7:11 the Primary 4th Edition [46]; for children older than 8:0 years the Intermediate 4th Edition [47]). Both TOLD versions measure several aspects of spoken language that are divided into four dimensions (semantics and grammar, listening, organizing, and speaking). All participants had a composite language score within the normal range (i.e., a scaled score of 85 or above). Children with TD and children with HFA did not differ significantly regarding their TONI and TOLD scores (see Table 1).

2.2.2. Pragmatic Language Measure: CCC-2. The child's caregiver was asked to complete the Children's Communication Checklist-2 (CCC-2) [14]. CCC-2 data is missing from two children, as two caregivers in the TD group did not complete the questionnaire even after several reminders. The 70-item questionnaire is divided into ten scales: four language structure scales (speech, syntax, semantics, and coherence), four pragmatic scales (inappropriate initiation, scripted language, use of context, and nonverbal communication), and two autism scales (social relations and interests). The caregiver rates each item on a scale from 0 to 3 indicating how often they have observed certain behaviors in their child: never, 
TABLE 1: Means and standard deviations of typically developing children (TD, $N=17)$ and children with high-functioning autism (HFA, $N=7$ ): age, TOLD-P4/I4, TONI-3, and CCC-2 scores (sum of scaled pragmatic scores). Group means compared with independent two-tailed $t$-tests.

\begin{tabular}{lccr}
\hline & Children with HFA & Children with TD & Group difference \\
& Mean (SD), range & Mean (SD), range & n.s. \\
Age in months & $93.6(9.33), 85-113$ & $98.1(5.41), 87-107$ & n.s. \\
TOLD standard score & $102.4(14.56), 85-122$ & $106.1(11.09), 87-129$ & n.s. \\
TONI standard score & $102.0(8.66), 97-121$ & $106.7(19.25), 85-150$ & $p<.001$ \\
CCC-2 pragmatic composite & $21.0(6.90), 12-29$ & ${ }^{*} 44.7,(9.48), 24-56$ & \\
\hline
\end{tabular}

${ }^{*}$ Missing data from two participants.

occasionally, frequently, or always. The scaled scores of the pragmatic scales (based on 28 items) were summed to form a pragmatic language composite in order to get a measure of pragmatic ability, where a lower score indicates more pragmatic difficulties [48]. For examples of items from the pragmatic scales, see Appendix A. As expected, children with HFA had significantly lower pragmatic scores than children with TD, $t(15.914)=6.64$ and $p<.001$; see Table 1 .

2.3. Procedure. Each child met individually with a trained research assistant (a graduate speech-language pathology student) in a quiet room at the child's school. The child participated in two sessions, approximately 45 minutes each, which included a structured conversational language task (described below), the battery of standardized tests, and other discourse tasks unrelated to the present study. The structured conversational task was conducted at the beginning of the first session. The children were video-recorded for the duration of the sessions.

2.4. Conversational Language Sample. The structured conversational task required each child to talk for approximately 10 minutes with the experimenter, following a protocol with interview questions about topics including family, interests, and events, as well as some open-ended questions in an attempt to control for variation in conversational content. The experimenter provided a model by sharing information about, for example, her family and favorite things and then asked the child to do the same. The experimenter was allowed to give as much verbal and nonverbal support as needed, and focus was on creating a natural conversational context while at the same time eliciting a sufficiently long language sample from the child. A similar protocol has been suggested by Hadley [49]. Similar protocols were also used by Losh and Capps [21] and Goldman [20] to elicit personal narratives, although we define this as a conversational task since there were no constraints on the experimenter to refrain from giving normal conversational feedback. The experimenter was unaware of the measures for analysis.

2.4.1. Transcription and Segmenting. Language samples were transcribed orthographically by the first and second authors and by three trained research assistants and analyzed using the software SALT (Systematic Analysis of Language Transcripts) [17]. The transcriptions were segmented into both communication units (C-units) and phonological units (Punits), following the rules specified in the SALT manual [50].

$\mathrm{C}$-units and P-units are two different methods of segmenting a transcript into utterances. Segmentation into Cunits is based on grammatical rules, where each C-unit contains one independent clause and all its dependent clauses, which is in contrast to P-units, where intonation and pauses are used to determine the completion of an utterance. C-units reflect grammatical complexity to a higher extent and have been shown to be more reliable across transcribers, while Punits reflect the actual length of a spoken utterance to a larger extent [50]. In the current study, C-units were used for the analysis of causal language since independent causal language increases grammatical complexity, and P-units were used for speech disruptions since the length of utterance has been shown to affect the amount of speech disruptions in several previous studies (e.g., $[5,7,35])$.

For all analyses, elliptical and imitative responses, as well as yes-no replies, were excluded so that these types of short utterances did not deflate the mean utterance length (MLPU2) [51].

2.4.2. Causal Language Analysis. The measures included in the causal language analyses were clausal density and independent and solicited causal statements. Clausal density was included as a direct measure of syntactic complexity. Clausal density is the total number of clauses (main + dependent) per main clause. Main and dependent clauses were coded to calculate density, following the procedure used by Nippold et al. [52]; see Appendix B. Causal language was defined as statements where the child conveyed the reason, cause, or motivation for an event or emotion, using the conjunctions so or because [26]; see Appendix C. Furthermore, all causal statements were divided into two types: independent, where causal language is used spontaneously and independently by the child, and solicited, where causal statements follow a prompt (a why-question [26]). Why-questions from the examiner were also coded, to ensure that both groups of children received a similar amount of why-prompts during the conversation. In order to compare causal language between children and control for transcript length, independent and solicited causal statements were expressed per C-unit.

2.4.3. Speech Disruption Analysis. The measures included in the analyses of speech disruptions were MLPU2, revisions and repetitions, filled pauses, and total speech disruptions. 
TABLE 2: Descriptive data for number of C-units, clausal density, and causal language of children with HFA $(N=7)$ and children with TD $(N=17)$.

\begin{tabular}{|c|c|c|c|c|}
\hline & \multicolumn{2}{|c|}{ Children with HFA } & \multicolumn{2}{|c|}{ Children with TD } \\
\hline & Mean (SD) & Range & Mean (SD) & Range \\
\hline Total number of C-units & $80.9(20.13)$ & $43-128$ & $99.3(30.24)$ & $51-136$ \\
\hline Clausal density & $1.15(0.0792)$ & $1.03-1.27$ & $1.22(0.0801)$ & $1.07-1.33$ \\
\hline${ }^{*}$ Independent causal statements/C-unit & $0.013(0.010)$ & $0-0.02$ & $0.064(0.046)$ & $0.01-0.18$ \\
\hline Solicited causal statements/C-unit & $0.014(0.010)$ & $0-0.03$ & $0.023(0.016)$ & $0-0.06$ \\
\hline Number of "why" questions & $2.00(1.414)$ & $1-4$ & $1.82(1.131)$ & $0-4$ \\
\hline
\end{tabular}

* Significant difference between groups, $p<.001$.

Speech disruptions were divided into two categories: revisions/repetitions and filled pauses [39]. The transcription and coding of speech disruptions followed the standardized procedures described in the SALT software manual, where any sequence of speech disruptions is enclosed in parentheses and not included in the subsequent calculation of MLPU2, and filler words (e.g., "like") are manually marked with the code [FW] [50]. The SALT software automatically quantifies the number and type of speech disruptions (fillers, repetitions, and revisions). In order to compare the number of speech disruptions between children while controlling for transcript length, all speech disruption measures were expressed per P-unit.

2.4.4. Reliability. Reliability testing was carried out in three steps: reliability of transcription, reliability of segmentation in C-units and P-units, and reliability of coding.

$16 \%$ of the recordings were retranscribed, and interrater reliability was determined by number of words in agreement, including all speech disruptions. Agreement was computed by dividing the total number of words in agreement by the total number of words in the transcript. Average agreement was $95.4 \%$ (range 92.9-97.9\%). All disagreements in the reliability transcriptions were solved through discussion. A graduate student, who was blind to the diagnostic status of the children, segmented the transcripts in C-units and P-units and calculated MLPU2. She also recoded those transcripts for clausal density, causal language, and examiner prompts for causal language. Reliability for number of C-units was $98.6 \%$ and number of P-units was $96.7 \%$, calculated as percent agreement on raw numbers. Correlations showed that reliability of MLPU2 was $r=0.974$, of clausal density was $r=0.928$, and of causal language was $r=0.935$. Reliability of coding why-prompts from the experimenter reached $100 \%$.

\section{Results}

SPSS 23 was used for all statistical analyses. Independent samples $t$-tests assuming unequal variances were performed to make group comparisons between the children with HFA and the children with TD. Cohen's $d$, using pooled standard deviations, was calculated as effect size estimates. Pearson bivariate and partial correlations were used to examine the relationships between pragmatic language skills and causal language or speech disruptions. For the correlations, scatterplots were examined and the relationships between the variables of interest were found to be linear, without significant outliers. Assumptions of normality and homoscedasticity were checked through boxplots and residual plots and were found to be sufficiently met. Since only a small number of planned comparisons and correlations were made, the overly conservative Bonferroni-correction was not applied, since that would highly inflate the risk of making type-II errors [53].

\subsection{Group Differences}

3.1.1. Causal Language. Before analyzing causal language use, we examined the length (complete and intelligible C-units) of the language samples of the two groups (see Table 2). There was no significant difference in number of $\mathrm{C}$-units between groups, $t(8.923)=-1.54$ and $p=.157$.

To investigate differences in the use of causal language, we examined clausal density, independent and solicited causal statements, and number of "Why" questions from the experimenter. Means and standard deviations of all measures are presented in Table 2.

There were no significant differences between children with HFA and children with TD in clausal density, $t(11.365)=2.03$ and $p=.066$, solicited causal statements/Cunit, $t(18.037)=1.63$ and $p=.121$, or the number of "Why" questions, $t(9.334)=-0.29$ and $p=.775$. However, as predicted, children with HFA used significantly less independent causal statements/C-unit than children with TD, $t(18.95)=4.35, p<.001$, and $d=1.532$.

3.1.2. Speech Disruptions. To investigate group differences in the use of speech disruptions, we examined MLPU2, revisions and repetitions per P-unit, and filled pauses per P-unit. Means and standard deviations of all measures are presented in Table 3. Children with HFA and children with TD did not differ significantly on MLPU2, $t(9.840)=2.226$ and $p=.051$, or revisions and repetitions, $t(20.503)=1.68$ and $p=.107$, but children with HFA had a significantly lower proportion of filled pauses than children with TD $[t(14.209)=2.27$, $p=.039]$, which was according to the hypothesis.

3.2. Relationships with Pragmatic Skills. CCC-2 scores were missing for two of the children in the TD group; hence, 
TABLE 3: Descriptive data for mean length of P-unit, revisions/repetitions, and filled pauses for children with HFA $(N=7)$ and children with $\operatorname{TD}(N=17)$.

\begin{tabular}{|c|c|c|c|c|}
\hline & \multicolumn{2}{|c|}{ Children with HFA } & \multicolumn{2}{|c|}{ Children with TD } \\
\hline & Mean (SD) & Range & Mean (SD) & Range \\
\hline MLPU2 & $7.61(2.331)$ & $5.40-10.98$ & $9.85(1.998)$ & $6.96-13.46$ \\
\hline Revisions and repetitions/P-unit & $0.36(0.191)$ & $0.06-0.56$ & $0.55(0.371)$ & $0.05-1.36$ \\
\hline${ }^{*}$ Filled pauses/P-unit & $0.23(0.205)$ & $0.03-0.59$ & $0.46(0.259)$ & $0.09-1.11$ \\
\hline
\end{tabular}

${ }^{*}$ Significant difference between groups, $p=.039$.

TABLE 4: Partial correlations between filled pauses, revisions/repetitions, MLPU2, and pragmatic scales. TOLD composite language scores are included as a covariate $(n=22)$.

\begin{tabular}{|c|c|c|c|}
\hline & Sum of pragmatic scales & Filled pauses per P-unit & Revisions and repetitions per P-unit \\
\hline \multicolumn{4}{|c|}{ Sum of CCC- 2 pragmatic scales } \\
\hline Correlation $(r)$ & & $.460^{*}$ & .216 \\
\hline$p$ value & & .036 & .348 \\
\hline \multicolumn{4}{|c|}{ Mean length of P-unit (MLPU2) } \\
\hline Correlation $(r)$ & .401 & $.621^{* *}$ & $.746^{* * *}$ \\
\hline$p$ value & .072 & .003 & .000 \\
\hline
\end{tabular}

${ }^{*} p<.05,{ }^{* *} p<.005$, and ${ }^{* * *} p<.001$.

the total number of participants included in correlations involving CCC- 2 scores was 22 (7 children with HFA, 15 with TD).

3.2.1. Causal Language. A correlation between pragmatic scores and the number of independent causal statements per C-unit including all children yielded a small positive, but insignificant relationship, $r=0.303$ and $p=.170$, which did not support the hypothesis.

3.2.2. Speech Disruptions. To investigate the relationship between speech disruptions, MLPU2, TOLD composite score, and pragmatic scores, a series of bivariate correlations were carried out.

As expected, MLPU2 was significantly positively correlated with both filled pauses $(r=.655, p=.001)$ and repetitions/revisions $(r=.588, p=.004)$, indicating that as utterance length increases, both categories of speech disruptions also increase.

Pragmatic scores were significantly and positively correlated with filled pauses $(r=.517, p=.014)$ but not with repetitions/revisions $(r=.110, p=.625)$. Pragmatic scores were also significantly and positively related to the TOLD composite score, however $(r=0.479, p=.024)$. To ensure that the relationship between pragmatic scores and filled pauses was not driven by underlying differences in TOLD scores between participants, the TOLD composite was included as a covariate in a new series of correlations between speech disruptions, MLPU2, and the pragmatic scores.

Table 4 shows the partial correlations after controlling for TOLD composite score. As expected, MLPU2 is still significantly correlated to both proportion of filled pauses and proportion of revisions and repetitions. Importantly, however, the pragmatic scores continue to be significantly related to filled pauses but not to revisions/repetitions or
MLPU2, a finding that is according to the hypothesis. These results indicate different functions of filled pauses and revisions and repetitions in conversation, which will be further addressed in the discussion.

\section{Discussion}

This study investigated causal language and filled pauses in conversation in school-age children with high-functioning autism (HFA) and typical development (TD) and examined the relationship between pragmatic ability and these structural aspects of language. All children scored within normal range of standardized verbal and nonverbal tests, and groups did not differ significantly in age, composite language scores, nonverbal IQ, or language sample complexity. The differences between groups were therefore interpreted as a result of differences in pragmatic ability. To the authors' knowledge, this is the first study that has found significant relationships while explicitly controlling for and reporting relevant structural language skills.

4.1. Causal Language. The results confirmed the first hypothesis: children with HFA showed less independent causal statements but a similar amount of solicited causal statements. This indicates that children with HFA provide the listener with solicited explanations to the same degree as their TD peers. In the absence of an explicit prompt, however, they tend to provide explanations to a lesser extent. The underlying reason might be a lower sensitivity to listener needs and/or an inability to follow cultural communicative conventions [25]. These results are in line with several previous studies including children with HFA $[18,21,23,27,28]$. The conclusion that there is a relationship between pragmatic ability and causal language use is strengthened by the lack of significant differences between groups in clausal density of the conversational 
sample and standardized language test scores. How speakers use language in different communicative situations is highly context dependent, however. Speakers are challenged in different ways by a range of contextual aspects, such as conversational topic and speaking partner. Variability within groups is therefore fairly common, particularly in studies of children with any type of communication disorder. In the present study, there was considerable within-group variability in proportion of causal statements in both groups of children. Even though the experimenter followed the same protocol for all children, the focus was to create a natural conversational context, and the content of the conversation was therefore not strictly controlled. The length of the language sample also varied in both groups of children, with the consequence that some children had fewer opportunities to include causal statements. Even though language sample length was factored into the analyses (by expressing causal statements per Cunit), these factors may have contributed to the variability. Although the design of this study does not allow for a thorough investigation of the underlying reason for less use of causal language in the group of children with HFA, the finding contributes to our understanding of some of their conversational patterns. Less use of independent causal language might tax the speaking partner more, because it requires inferring or trying to elicit information when the child does not provide it voluntarily. Such patterns may also be perceived as "different" and contribute to our impression of poor pragmatic skills, although these children have structural language skills that are within the normal range according to standardized testing and standard language sample analysis measures.

Although there was a significant difference between groups in the use of causal language, there was no significant relationship between pragmatic scores on the CCC-2 and independent causal language, which was not according to the hypothesis (a positive but nonsignificant relationship was found). It is possible that, with a larger group of children with HFA and increased power for statistical analysis, there might have been a significant relationship. It is also possible that the items in the CCC-2 quantify pragmatic ability more broadly, with the consequence that causal language is too specific and subtle of a feature of social/pragmatic ability to be linked to this measure. Development of complex language (including causal language) has been shown to be related to the development of theory of mind [54], and therefore a direct measure of theory of mind might has shown a stronger relationship to causal language use. Such a measure was not included in the present study but should be explored in future studies.

4.2. Speech Disruptions. The results confirmed the hypothesis that children with HFA would demonstrate a significantly lower proportion of filled pauses (but not of revisions/repetitions) compared to TD children in conversation. The group difference in mean length of P-unit also approached significance, however $(p=.051)$, which means that the significant group difference in proportion of filled pauses should be interpreted with care. After controlling for a potential spurious variable (TOLD composite score), a significant positive correlation was found between the pragmatic scores (CCC-2) and the proportion of filled pauses but not with proportion of revisions/repetitions, which was in line with the hypothesis. Importantly, both filled pauses and revisions/repetitions were also correlated with MLPU2. While the results suggest that there may be a relationship between pragmatic ability and filled pauses, both categories of speech disruptions also increase when utterance length increases. This relationship supports previous research focusing on the language processing perspective of speech disruptions $[5,7,33-35]$ but is also in line with the previous studies that have found different patterns of speech disruption occurrence in children with ASD and children with TD $[24,32,38$, $40,42]$. The present study differed in several important ways from these previous studies including children with ASD, however. Only Suh et al. [32] matched participants on MLU and found a nonsignificant difference $(p=.07)$ between groups in terms of filled pauses, a result that could be due to the use of a narrative context rather than a conversational context. Previous studies have also identified fillers differently compared to the current study. All studies mentioned above (except [24]) included the filled pauses $u h$ and $u m$ in their analyses. In the present study, we included both filled pauses and filler words (content-empty words such as like and so), according to a classification proposed by Thordardottir and Weismer [39]. Even though a distinction between different types of filled pauses has been made previously $[24,36]$, we argue that all fillers may serve the same function: to hold the floor in conversation and signal to the listener that the speaker is not finished with his/her turn. If a speaker does not fill a pause, the result is a silent pause, which Lake et al. [38] found to be more prevalent in adults with HFA than in adults without HFA. An analysis of silent pauses would therefore be an interesting addition but was not included in the current study after initial analyses showed that it was difficult to reach high enough interrater reliability.

Importantly, even after controlling for structural language ability as measured by the TOLD, the strong and positive relationship between MLPU2 and both fillers and revisions/repetitions held. This indicates that it is unlikely that fillers only serve a pragmatic function. Children who speak in short utterances may therefore exhibit fewer fillers without this feature being associated with any pragmatic difficulties: with a shorter utterance there is less need to signal to the listener that the speech turn is not finished yet.

\subsection{Limitations and Future Directions. This study was limited} in sample size, but the analyses and matching between groups (both regarding age and structural language ability) were more rigorous than most previous studies. Further replication is needed to draw any firm conclusions, however. Conversation was judged to be the most appropriate speaking context to investigate the relationship between pragmatic ability and the chosen features of language production, but a more challenging speaking context might be included in future studies. Such contexts may include multiple speakers or tasks with persuasive or argumentative language. These contexts may tax children more, with a more explicit demand 
for holding the floor and taking several listeners' needs into account. They may therefore be even more revealing from the perspective of studying language production from a pragmatic perspective.

The present study only investigated a few aspects of structural language and the relationship to pragmatic skills and did not investigate the content of the conversation. The effect of different content could be revealing, however, since children with ASD often have restricted interests. The interaction between conversational content and features of speech and language form may be quantified and investigated by focusing on repetitive topics in language samples. A recent study by Rouhizadeh et al. [55] analyzed repetitive topics in conversational samples from children with ASD through an automatized method and found more overlap in terms of semantics in children with ASD compared to children with TD. This is an interesting route and could be done in conjunction with analyses of structural language skills and the pragmatic markers presented in the current study.

Finally, other groups of children also show pragmatic difficulties, including children with social communication disorder [56] and individuals within the wider autism spectrum. Future research could extend the present study to include these other groups to explore whether the differences in causal language and the use of fillers in conversation are specific for children with pragmatic difficulties due to autism.

\section{Conclusions}

The results of this study indicate that children with HFA use significantly fewer independent causal statements and exhibit fewer fillers in conversation compared to peers with TD, in spite of equally strong performance on standardized language tests and similar grammatical complexity/MLU. This adds to the previous literature, which has found similar relationships, but has failed to control for structural language abilities. Research should continue to focus on dialogic naturalistic contexts instead of language tests, as language sampling from naturalistic contexts increases our understanding of how these children make use of language for purposeful communication. The difficulties that children with HFA have in conversation might be subtle, and standard LSA measures might not capture the characteristics, even though the quality of the interaction might be perceived as "different." Less use of independent causal language places the burden of communication on the speaking partner, by requiring them to infer information or ask questions to a larger extent. Similarly, fewer fillers in conversation (with a possible increase in silent pauses as a consequence) may also make the natural back-and-forth in conversation more effortful for the typical conversational partner. A listener may perceive these characteristics as odd, even though the speaker's structural language ability is within normal range. Thus, these findings may be used to increase our understanding of how children with HFA may behave in conversation and provide two measures that would be easy to implement in clinical practice. Causal language might also be a possible target in language intervention, with a focus on causal explanations in social discourse, as well as in personal narratives. Such approaches could possibly be carried out in tandem with a focus on increased self-awareness and awareness of listener needs in order to strengthen theory of mind skills [54].

\section{Appendix}

\section{A. Examples of Items from the Pragmatic Scales of the CCC-2 [14]. Two Examples Refer to Difficulties (\#14, \#48) and Two Examples Refer to Strengths (\#56, \#60)}

\author{
Item \# Statement for the parent to rate \\ 14 \\ Does not look at the person he or she is \\ talking to. \\ Does not explain what he or she is talking \\ about to someone who does not share his \\ or her experiences (e.g., talks about "Johnny" \\ without explaining who Johnny is). \\ Makes good use of gestures to get his or her \\ meaning across. \\ Realizes the need to be polite (e.g., would \\ act pleased if given a present he or she did \\ not really like; would avoid making personal \\ comments about strangers).
}

\section{B. Calculation of Clausal Density $[4,52]$}

B.1. Main (Independent) Clause. A main clause consists of a subject and a main verb and makes a complete statement. For example, the following utterances are both independent clauses: "I met a new friend today" and "He runs very fast through the forest."

B.2. Subordinate (Dependent) Clause. A dependent clause contains a subject and a main verb but does not make a complete statement (i.e., cannot stand alone). For example, "...who was singing in the shower." There are three main types of subordinate clauses: nominal, adverbial, and relative. In the present study, all dependent clauses were coded and counted together.

B.3. Clausal Density. Clausal density is calculated by adding all main and subordinate clauses in the sample and dividing this number by the total number of main clauses. The number obtained reflects the degree of subordination in the sample. An example:

$$
\begin{aligned}
& \text { Main clauses }=102, \text { Subordinate clauses }=47 \rightarrow \\
& \text { Total clauses }=149 \\
& \text { Clausal density }=149 / 102=1.46
\end{aligned}
$$

\section{Causal Language Codes}

All codes were placed at the end of an utterance. One utterance could not receive more than one causal language code. All instances of child causal statements including "so" or "because" and experimenter "why"-questions were coded. 
C.1. Independent Causal Statement [CAU]. An independent causal statement is a statement used without a soliciting question.

Examples

C: I like going to the beach because I like playing in the sand [CAU].

C: She wanted to go to the mall so she could buy a new doll [CAU].

C.2. Why-Questions [WHY] and Solicited Causal Statements [SCAU]. A solicited causal statement follows a why-prompt from the experimenter, and both the prompt and the causal statement are coded.

Examples

C: I like dogs.

E: Why do you like dogs [WHY]?

C: Because they are fluffy [SCAU].

\section{Competing Interests}

The authors declare that there are no competing interests regarding the publication of this paper.

\section{Authors' Contributions}

Christina Reuterskiöld initially conceived the study, Anna Eva Hallin selected the features for analysis, collected and analyzed data, and wrote the manuscript together with Gabrielle D. Garcia, who also conducted part of the analyses. Christina Reuterskiöld participated in all of the processes through supervision, discussion, and review.

\section{Acknowledgments}

The authors express their gratitude to the children and parents who participated in the study from the schools in the NYU Steinhardt/New York City Department of Education ASD Nest Program and the Research Assistants in the CSD Department Small Talk Child Language Lab. The authors also extend their gratitude to Dr. Harriet Klein, Dr. Diana Sidtis, Dr. Susanne Quadflieg, and Dr. Diego Almeida for their comments on previous drafts of this paper. This study was supported by an NYU Steinhardt School Faculty Research Challenge Grant.

\section{References}

[1] G. Conti-Ramsden and K. Durkin, "Language development and assessment in the preschool period," Neuropsychology Review, vol. 22, no. 4, pp. 384-401, 2012.

[2] M. Dunn, J. Flax, M. Sliwinski, and D. Aram, "The use of spontaneous language measures as criteria for identifying children with specific language impairment: an attempt to reconcile clinical and research incongruence," Journal of Speech, Language, and Hearing Research, vol. 39, no. 3, pp. 643-654, 1996.
[3] L. H. Price, S. Hendricks, and C. Cook, "Incorporating computer-aided language sample analysis into clinical practice," Language, Speech, and Hearing Services in Schools, vol. 41, no. 2, pp. 206-222, 2010.

[4] M. A. Nippold, T. C. Mansfield, J. L. Billow, and J. B. Tomblin, "Expository discourse in adolescents with language impairments: examining syntactic development," American Journal of Speech-Language Pathology, vol. 17, no. 4, pp. 356-366, 2008.

[5] D. Wetherell, N. Botting, and G. Conti-Ramsden, "Narrative in adolescent specific language impairment (SLI): a comparison with peers across two different narrative genres," International Journal of Language \& Communication Disorders, vol. 42, no. 5, pp. 583-605, 2007.

[6] C. Reuterskiöld, K. Hansson, and B. Sahlén, "Narrative skills in Swedish children with language impairment," Journal of Communication Disorders, vol. 44, no. 6, pp. 733-744, 2011.

[7] C. Reuterskiöld Wagner, U. Nettelbladt, B. Sahlén, and C. Nilholm, "Conversation versus narration in pre-school children with language impairment," International Journal of Language \& Communication Disorders, vol. 35, no. 1, pp. 83-93, 2000.

[8] L. Stirling, S. Douglas, S. Leekam, and L. Carey, "The use of narrative in studying communication in Autism Spectrum Disorders," in Communication in Autism, J. Arciuli and J. Brock, Eds., vol. 11 of Trends in Language Acquisition Research, chapter 8, pp. 169-216, John Benjamins Publishing Company, Amsterdam, The Netherlands, 2014.

[9] E. Prud'hommeaux and M. Rouhizadeh, "Automatic detection of pragmatic deficits in children with autism," in Proceedings of the Workshop on Child, Computer and Interaction (WOCCI '12), pp. 1-6, Portland, Ore, USA, September 2012.

[10] I. Rapin, M. A. Dunn, D. A. Allen, M. C. Stevens, and D. Fein, "Subtypes of language disorders in school-age children with autism," Developmental Neuropsychology, vol. 34, no. 1, pp. 66$84,2009$.

[11] R. Paul, S. M. Orlovski, H. C. Marcinko, and F. Volkmar, "Conversational behaviors in youth with high-functioning ASD and Asperger syndrome," Journal of Autism and Developmental Disorders, vol. 39, no. 1, pp. 115-125, 2009.

[12] E. Rubin and L. Lennon, "Challenges in social communication in Asperger syndrome and high-functioning autism," Topics in Language Disorders, vol. 24, no. 4, pp. 271-285, 2004.

[13] C. D. Jones and I. S. Schwartz, "When asking questions is not enough: an observational study of social communication differences in high functioning children with autism," Journal of Autism and Developmental Disorders, vol. 39, no. 3, pp. 432443, 2009.

[14] D. V. M. Bishop, Children's Communication Checklist-2, CCC-2, PsychCorp, San Antonio, Tex, USA, 2003.

[15] J. Volden and L. Phillips, "Measuring pragmatic language in speakers with autism spectrum disorders: comparing the children's communication checklist- 2 and the test of pragmatic language," American Journal of Speech-Language Pathology, vol. 19, no. 3, pp. 204-212, 2010.

[16] C. Adams, "Practitioner review: the assessment of language pragmatics," Journal of Child Psychology and Psychiatry and Allied Disciplines, vol. 43, no. 8, pp. 973-987, 2002.

[17] J. Miller and A. Iglesias, Systematic Analysis of Language Transcripts (SALT), SALT Software, 2012. 
[18] L. Capps, M. Losh, and C. Thurber, “"The frog ate the bug and made his mouth sad': narrative competence in children with autism," Journal of Abnormal Child Psychology, vol. 28, no. 2, pp. 193-204, 2000.

[19] E. C. Young, J. J. Diehl, D. Morris, S. L. Hyman, and L. Bennetto, "The use of two language tests to identify pragmatic language problems in children with autism spectrum disorders," Language, Speech, and Hearing Services in Schools, vol. 36, no. 1, pp. 62-72, 2005.

[20] S. Goldman, "Brief report: narratives of personal events in children with autism and developmental language disorders: unshared memories," Journal of Autism and Developmental Disorders, vol. 38, no. 10, pp. 1982-1988, 2008.

[21] M. Losh and L. Capps, "Narrative ability in high-functioning children with autism or Asperger's Syndrome," Journal of Autism and Developmental Disorders, vol. 33, no. 3, pp. 239-251, 2003.

[22] C. F. Norbury and D. V. M. Bishop, "Narrative skills of children with communication impairments," International Journal of Language \& Communication Disorders, vol. 38, no. 3, pp. 287313, 2003.

[23] J. J. Diehl, L. Bennetto, and E. C. Young, "Story recall and narrative coherence of high-functioning children with autism spectrum disorders," Journal of Abnormal Child Psychology, vol. 34, no. 1, pp. 87-102, 2006.

[24] C. A. Irvine, I.-M. Eigsti, and D. A. Fein, "Uh, um, and autism: filler disfluencies as pragmatic markers in adolescents with optimal outcomes from autism spectrum disorder," Journal of Autism and Developmental Disorders, vol. 46, no. 3, pp. 10611070, 2016.

[25] M. Bamberg and R. Damrad-Frye, "On the ability to provide evaluative comments: further explorations of children's narrative competencies," Journal of Child Language, vol. 18, no. 3, pp. 689-710, 1991.

[26] M. Lahey, "Language disorders and language development," in Language Development and Language Disorders/Lois Bloom, Margaret Lahey, 1978, Macmillan, Upper Saddle River, NJ, USA, 1988.

[27] R. B. Beaumont and P. Newcombe, "Theory of mind and central coherence in adults with high-functioning autism or Asperger syndrome," Autism, vol. 10, no. 4, pp. 365-382, 2006.

[28] H. Tager-Flusberg, “'Once upon a ribbit': stories narrated by autistic children," British Journal of Developmental Psychology, vol. 13, no. 1, pp. 45-59, 1995.

[29] S. Baron-Cohen, A. M. Leslie, and U. Frith, "Mechanical, behavioural and intentional understanding of picture stories in autistic children," British Journal of Developmental Psychology, vol. 4, no. 2, pp. 113-125, 1986.

[30] L. Colle, S. Baron-Cohen, S. Wheelwright, and H. K. J. Van Der Lely, "Narrative discourse in adults with high-functioning autism or Asperger syndrome," Journal of Autism and Developmental Disorders, vol. 38, no. 1, pp. 28-40, 2008.

[31] H. Tager-Flusberg and K. Sullivan, "Attributing mental states to story characters: a comparison of narratives produced by autistic and mentally retarded individuals," Applied Psycholinguistics, vol. 16, no. 3, pp. 241-256, 1995.

[32] J. Suh, I.-M. Eigsti, L. Naigles, M. Barton, E. Kelley, and D. Fein, "Narrative performance of optimal outcome children and adolescents with a history of an Autism Spectrum Disorder
(ASD)," Journal of Autism and Developmental Disorders, vol. 44, no. 7, pp. 1681-1694, 2014.

[33] W. J. M. Levelt, Speaking: From Intention to Articulation, The MIT Press, Cambridge, Mass, USA, 1993.

[34] M. Rispoli, P. Hadley, and J. Holt, "Stalls and revisions: a developmental perspective on sentence production," Journal of Speech, Language, and Hearing Research, vol. 51, no. 4, pp. 953966, 2008.

[35] B. G. MacLachlan and R. S. Chapman, "Communication breakdowns in normal and language learning-disabled children's conversation and narration," The Journal of Speech and Hearing Disorders, vol. 53, no. 1, pp. 2-7, 1988.

[36] H. H. Clark and J. E. F. Tree, "Using $u h$ and $u m$ in spontaneous speaking," Cognition, vol. 84, no. 1, pp. 73-111, 2002.

[37] H. H. Clark, "Speaking in time," Speech Communication, vol. 36, no. 1-2, pp. 5-13, 2002.

[38] J. K. Lake, K. R. Humphreys, and S. Cardy, "Listener vs. speaker-oriented aspects of speech: studying the disfluencies of individuals with autism spectrum disorders," Psychonomic Bulletin \& Review, vol. 18, no. 1, pp. 135-140, 2011.

[39] E. T. Thordardottir and S. E. Weismer, "Content mazes and filled pauses in narrative language samples of children with specific language impairment," Brain and Cognition, vol. 48, no. 2-3, pp. 587-592, 2002.

[40] P. A. Heeman, R. Lunsford, E. Selfridge, L. Black, and J. van Santen, "Autism and interactional aspects of dialogue," in Proceedings of the 11th Annual Meeting of the Special Interest Group on Discourse and Dialogue (SIGDIAL '10), pp. 249252, The University of Tokyo: Association for Computational Linguistics, Bunkyō, Japan, September 2010.

[41] S. F. Epstein, Formulation Deficits in Spontaneous Speech of Verbal Children with High Functioning Autism, Graduate School of Arts and Sciences, Columbia University, 2005.

[42] D. Bone, C.-C. Lee, T. Chaspari et al., "Acoustic-prosodic, turntaking, land langauge cues in child-psychologist interactions for varying social demand," in Proceedings of the 14th Annual Conference of the International Speech Communication Association (INTERSPEECH '13), pp. 2400-2404, International Speech Communication Association, Lyon, France, August 2013.

[43] C. Lord, S. Risi, L. Lambrecht et al., "The autism diagnostic observation schedule-generic: a standard measure of social and communication deficits associated with the spectrum of autism," Journal of Autism and Developmental Disorders, vol. 30, no. 3, pp. 205-223, 2000.

[44] H. Sturm, E. Fernell, and C. Gillberg, "Autism spectrum disorders in children with normal intellectual levels: associated impairments and subgroups," Developmental Medicine \& Child Neurology, vol. 46, no. 7, pp. 444-447, 2004.

[45] L. Brown, R. Sherbenou, and S. Johnsen, TONI-3: Test of Nonverbal Intelligence Examiner's Manual, PRO-ED Inc, Austin, Tex, USA, 1997.

[46] P. Newcomer and D. D. Hammill, Test of Language Development: Primary, Pro-Ed, Austin, Tex, USA, 4th edition, 2008.

[47] D. D. Hammill and P. Newcomer, Test of Language Development: Intermediate, Pro-Ed, Austin, Tex, USA, 4th edition, 2008.

[48] A. Philofsky, D. J. Fidler, and S. Hepburn, "Pragmatic language profiles of school-age children with autism spectrum disorders and Williams Syndrome," American Journal of Speech-Language Pathology, vol. 16, no. 4, pp. 368-380, 2007. 
[49] P. A. Hadley, "Language sampling protocols for eliciting textlevel discourse," Language, Speech, and Hearing Services in Schools, vol. 29, no. 3, pp. 132-147, 1998.

[50] J. F. Miller, K. Andriacchi, and A. Nockerts, Assessing Language Production Using SALT Software-A Clinician's Guide to Language Sample Anlysis, SALT Software, Middleton, Wis, USA, 2011.

[51] J. R. Johnston, "An alternate MLU calculation: magnitude and variability of effects," Journal of Speech, Language, and Hearing Research, vol. 44, no. 1, pp. 156-164, 2001.

[52] M. A. Nippold, L. J. Hesketh, J. K. Duthie, and T. C. Mansfield, "Conversational versus expository discourse: a study of syntactic development in children, adolescents, and adults," Journal of Speech, Language, and Hearing Research, vol. 48, no. 5, pp. 10481064, 2005.

[53] T. V. Perneger, "What's wrong with Bonferroni adjustments," The British Medical Journal, vol. 316, no. 7139, pp. 1236-1238, 1998.

[54] C. A. Miller, "Developmental relationships between language and theory of mind," American Journal of Speech-Language Pathology, vol. 15, no. 2, pp. 142-154, 2006.

[55] M. Rouhizadeh, R. Sproat, and J. van Santen, "Similarity measures for quantifying restrictive and repetitive behavior in conversations of autistic children," in Proceedings of the 2nd Workshop on Computational Linguistics and Clinical Psychology: From Linguistic Signal to Clinical Reality, pp. 117-123, Association for Computational Linguistics, Colorado, Canada, 2015.

[56] APA, Diagnostic and Statistical Manual of Mental Disorders: DSM-5, American Psychiatric Association, Washington, DC, USA, 2013. 


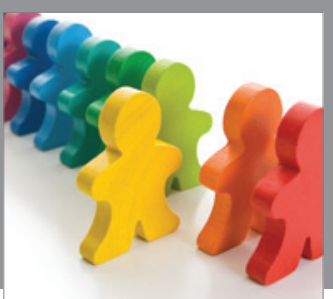

Autism

Research and Treatment
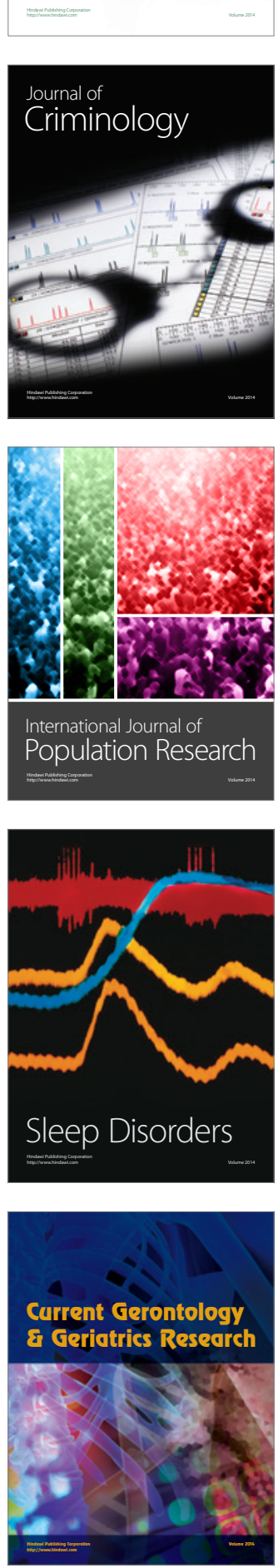

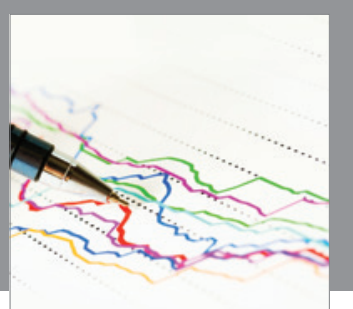

Economics

Research International
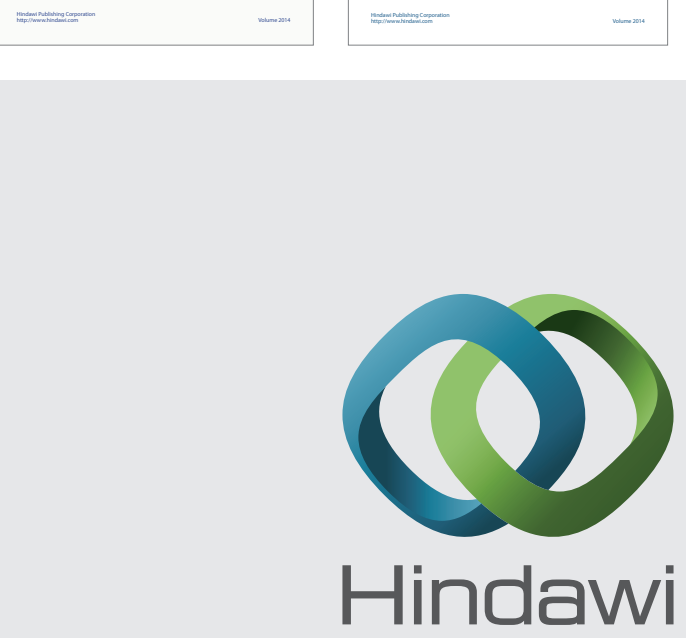

Submit your manuscripts at

http://www.hindawi.com
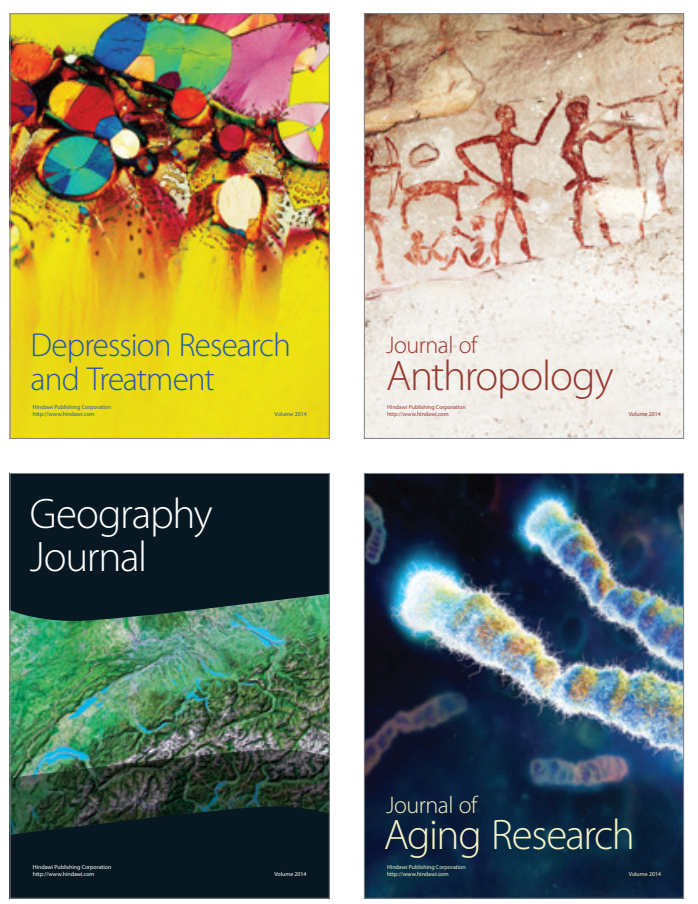
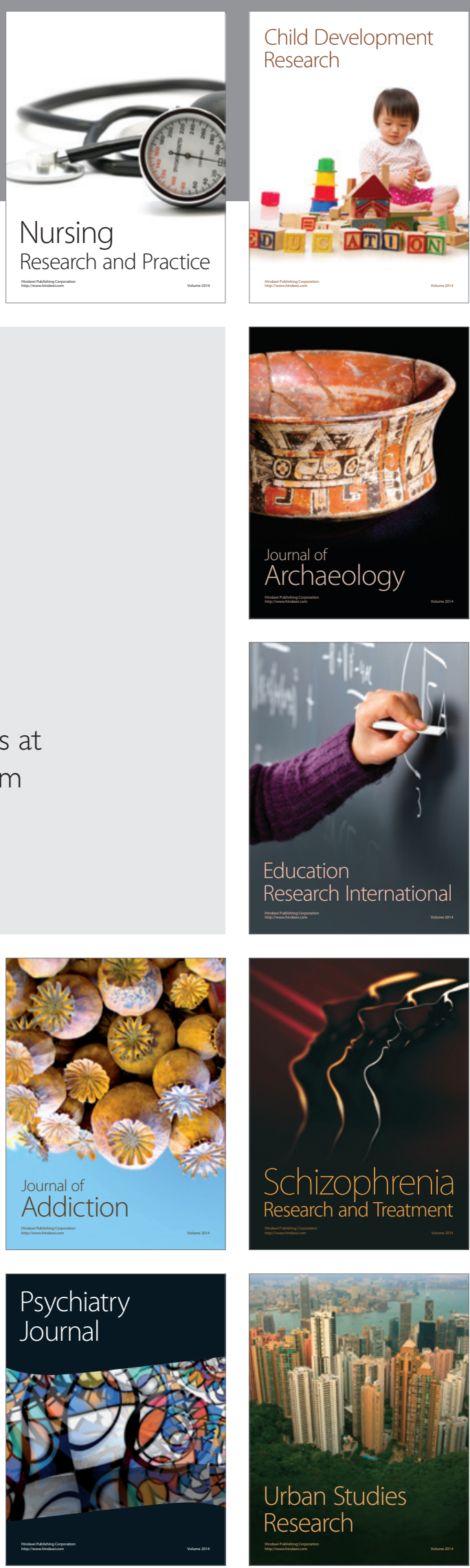